\section{Surgery for Low Back Pain}

M. Szpalski, R. Gunzburg, B.L. Rydevik, J.-C. Le Huec, H.M. Mayer (Eds) By Denis L. Kaech

This book is an interdisciplinary, multi-author approach to the low back pain problem; it is subdivided into six parts: Basics, Diagnosis, Conservative Treatment, Surgical Treatment (Fusion), Surgical Treatment (Other Technologies), and Outcomes.

After introducing the biology of disc degeneration, with an accent on genetic factors, the question "where does the pain come from" is discussed without conclusive answer, more research and better diagnostic tools being warranted in the future. The role of cytokines such as TNF and Interleukines is reviewed as well as various psychosocial aspects. The "instability" chapter recalls some controversies and insists on the importance of the pattern of motion (POM), rather than considering the angular ROM only, offering some illustrations of "animal and human in vitro testing".

No illustrations are found in the chapter about "Dynamic MRI of the Spine", that summarizes history and developments for assessing position and motion dependent dynamic changes within the spine, including references until 2007. A new endplate classification and score supplementing the Modic changes is presented, offering a more subtle and detailed assessment of MRI pictures, including diffusion following IV gadolinium injection. The role of physician extenders in a low back pain practice, as nurses, physiotherapists, certified athletic trainers, etc. is finally presented as a solution for shortening waiting periods and increasing the doctor's time for one-on-one consultations.

After an introduction about the natural evolution of non-specific low back pain, the conservative treatment section deals with the prescription of such treatments, including comprehensive rehabilitation and back schools, chiropractic care, as well as intradiscal electrothermal therapy
(IDET), and percutaneous intradiscal radiofrequency thermocoagulation (PIRFT). As the efficacy of these two techniques has not been proven by RCTs, the author concludes that clinicians should refrain from using these "minimally invasive" interventions to treat discogenic low back pain. Finally no hard scientific evidence from RCTs could be found to support the effectiveness of lumbar orthoses for prevention or treatment of non-specific low back pain. The main part of this book is dedicated to the various available surgical options and begins with a larger and well-illustrated chapter about the indications for lumbar spinal fusion. After a short and critical comment on the efficacy of pedicle based systems, a warning that low back pain (without demonstrated biomechanical instability) is not an indication for fusion after decompression for lumbar stenosis, the concept of hybrid construct is presented, followed by the management of spondylolysis and spondylolisthesis, supplemented by a chapter about the less known but cost saving transpedicular-transdiscal-transcorporal-fixation for one level SPL. The facet joint problems, and the adjacent level or segment disease (ASD) are further discussed, concluding that ASD should be considered as a fact. That it could be avoided using modern non-rigid devices is today however still a myth. Pelvic girdle pain, sacro-iliac joint problems, and blood loss management in major spine surgery are briefly discussed, followed by refining the indications for disc replacement in the treatment algorithm before indicating a fusion. Factors that may affect the outcome after lumbar TDR are discussed based on larger case studies, some with follow-up times of 10, 13 and even 17 years, followed by a smaller prospective randomized study showing that KineFlex and Charité lumbar TDR had similar results. This is followed by a chapter about the limitations of lumbar disc arthroplasty.

Six chapters are dedicated to posterior dynamic, "semi-rigid" or "non-rigid" stabilization, with finite element analysis, comparative biomechanical tests, overview of pedicle screw-based systems, a comparison of Dynesys, that does not perform better than fusion, completed by a review of problems and failures with non-rigid systems.

"Interspinous implants: State of the art and research of evidence" is a chapter about preliminary evidence with the InSwing device (preserving the supraspinous ligament tension band, prospective study with one year FU reporting marked clinical improvement of ODI and VAS, after prior cadaveric and in-vivo animal tests), with a summary of the literature until 2008 about other devices like Coflex, DIAM, Wallis and X-Stop. The next chapter presents the Nubac Disc Arthroplasty System, a PEEK implant mimicking the natural kinematics of an intact disc - a pre-clinical study.

The chapter dedicated to outcome assessment for cost-utility evaluation compares SF-6D versus EQ-SD. Both were found to be practicable and valuable, but not interchangeable for the estimation of QALY gain in case of poor health or major illness.

Finally there is a last contribution about the medical evidence regarding the surgical treatment of low back pain. Fortunately this chapter offers more: RCTs about fusion, TDR, the Cochrane studies about discectomy, the SPORT trails 20062008 are summarized and critically discussed. Patients with a definitive source of pain may benefit from surgery (e.g. unstable spondylolisthesis with stenosis), but not those with low back pain only. As the authors put it: "More research is needed to elucidate these uncertainties". To conclude, this book is a valuable contribution about lumbar spine problems, including basics, critical evaluation of different treatment options with EBM references, with brief ethical and socioeconomic discussions. Several chapters would deserve to be quoted in future publications. •

/+/ Surgery for Low Back Pain

2010, Hardcover, 285 pp, 69 illus., 29 in colour EUR (D) 106,95 CHF 143,50

Springer-Verlag, Berlin, Heidelberg ISBN: 978-3-642-04546-2 